\title{
Qualidade de vida dos idosos cadastrados no programa hiperdia de uma unidade básica de saúde na região norte
}

\author{
Quality of life of the elderly registered in the hiperdia program of a basic health unit in the \\ north region
}

Calidad de vida de los señores cadastrados en el programa hiperdia de una unidad básica de salud en la región norte

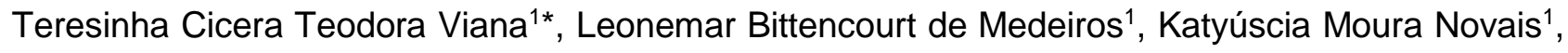
Alberto Luciano Gomes da Silva ${ }^{1}$, Simone Oliveira Pipper ${ }^{5}$, Marcélio Viana da Silva ${ }^{1}$

\section{RESUMO}

Objetivo: Identificar os níveis e a percepção de qualidade de vida entre os idosos cadastrados no Programa de Hiperdia no município de Cacoal- RO. Método: Foram entrevistados na unidade 58 idosos entre 60 e 86 anos de idade, sendo coletadas informações sobre gênero, idade, nível de escolaridade, estado civil e autoavaliação da saúde e utilizado os instrumentos WHOQOL-BREF e WHOQOL-OLD da Organização Mundial da Saúde para avaliar a qualidade de vida. Resultados: Houve uma predominância do sexo feminino entre os entrevistados, quanto à escolaridade a maior porcentagem está em relação ao ensino fundamental incompleto foi de $31(53,4 \%)$, analfabetismo $24(41,5 \%)$ e ensino médio $3(5,2 \%)$. A renda mensal da maioria é de 1 a 2 salários mínimos 46 (79,3\%). Em relação à limitação física, $12(20,7 \%)$ responderam que sim e 46 $(79,3 \%)$ responderam que não. No WHOQOL-BREF constatou que o nível de satisfação com a qualidade de vida encontrada para o domínio físico psicológico e ambiental foi médio, enquanto o domínio social apresentou o nível de satisfação alto. No WHOQOL-OLD apresenta o menor escore para as facetas autonomia e participação social com média de 3,5 e o maior escore morte e intimidade 3,9. Conclusão: A qualidade de vida entre a maioria dos idosos entrevistados apresenta um nível satisfatório, evidenciou uma avaliação positiva da mesma, principalmente em relação ao domínio social e psicológico, seguido dos domínios meio ambiente e físico, positivismo também quanto aos sentimentos de companheirismo e amor, as inquietações e temores com a morte.

Palavras chave: Idoso, Hiperdia, Qualidade de vida.

\begin{abstract}
Objective: To identify the levels and perception of quality of life among the elderly enrolled in the Hiperdia Program in the municipality of Cacoal-RO. METHODS: Fifty-eight individuals between 60 and 86 years of age were interviewed. Data on gender, age, schooling, marital status and health self-assessment were collected from WHOQOL-BREF and WHOQOL-OLD instruments to evaluate the quality of life. Results: There was a predominance of the feminine sex between the interviewed ones, as for the schooling the biggest percentage is regarding the incomplete basic teaching it was of $31(53,4 \%)$, illiteracy $24(41,5 \%)$ and secondary education $3(5,2 \%)$. The monthly income of the majority is from 1 to 2 minimum wages 46 (79,3\%). Regarding the physical limitation $12(20.7 \%)$ answered that yes and 46 (79.3\%) answered that they did not. The WHOQOLBREF found that the level of satisfaction with the quality of life found for the psychological and environmental
\end{abstract}

\footnotetext{
${ }^{1}$ Faculdade de Ciências Biomédicas de Cacoal -FACIMED. *E-mail: teresinhaenfermeira@hotmail.com

${ }^{2}$ Faculdade de Pimenta Bueno/ FAP
} 
physical domain was average, while the social domain had a high level of satisfaction. In the WHOQOL-OLD it presents the lowest score for the facets autonomy and social participation with a mean of 3.5 and the highest death and intimacy score 3.9. Conclusion: The quality of life among the majority of the elderly respondents presented a satisfactory level, evidenced a positive evaluation of the same, mainly in relation to the social and psychological domain, followed by the environment and physical domains, positivism also regarding the feelings of companionship and love, the restlessness and fears of death.

Keywords: Elderly, Hyperdia, Quality of life.

\section{RESUMEN}

Objetivo:Identificar los niveles y la percepción de calidad de vida entre los ancianos catastrados en el Programa de Hiperdia en el municipio de Cacoal- RO. Método: Se entrevistaron en la unidad 58 ancianos entre 60 y 86 años de edad, siendo recolectadas informaciones sobre género, edad, nivel de escolaridad, estado civil y autoevaluación de la salud y utilizado los instrumentos WHOQOL-BREF y WHOQOL-OLD de la Organización Mundial de la Salud para evaluar la calidad de vida. Resultados: Había un predominio del sexo femenino entre entrevistado, en cuanto a la educación el porcentaje más grande considera la enseñanza básica incompleta era del 31 (el 53,4\%), analfabetismo el 24 (el 41,5\%) y educación secundaria el 3 (el 5,2\%). Los ingresos mensuales de la mayoría son de 1 a 2 salarios mínimos el 46 (el 79,3\%). En el WHOQOL-BREF constató que el nivel de satisfacción con la calidad de vida encontrada para el dominio físico psicológico y ambiental fue medio, mientras que el dominio social presentó el nivel de satisfacción alto. En el WHOQOLOLD presenta el menor escore para las facetas autonomía y participación social con media de 3,5 y el mayor escore muerte e intimidad 3,9. Conclusión: La calidad de vida entre la mayoría de los ancianos entrevistados presenta un nivel satisfactorio, evidenció una evaluación positiva de la misma, principalmente en relación al dominio social y psicológico, seguido de los dominios medio ambiente y físico, positivismo también en cuanto a los sentimientos de compañerismo y amor, las inquietudes y temores con la muerte.

Palabras clave: Ancianos, Hiperdia, Calidad de vida.

\section{INTRODUÇÃO}

Segundo a Organização Mundial de Saúde (OMS, 2005) o Brasil será o sexto país com maior número de pessoas idosas até 2025. O crescimento da população idosa acontece de forma radical e rápida, de acordo com (VERAS, 2008) o número de idosos será superior a 30 milhões de pessoas, tornando-se urgente a necessidade de investigações que contribuam para melhoria e/ou manutenção da saúde e qualidade de vida nessa faixa etária. Diabetes mellitus, doenças pulmonares, doença vascular periférica, doenças valvares degenerativas, doenças renais, doenças hepáticas e depressão são comorbidades que atingem essa população e são um problema de saúde global, quando cuidadas poderão prevenir melhor as doenças e oferecer uma melhor qualidade de vida (ZASLAVSK e GUS, 2001).

Segundo Freitas (2002) os idosos vivem a velhice de forma diversificada, como se o fim da vida reproduzisse e ampliasse as desigualdades sociais. Com o aumento da população idosa em nossa sociedade, constitui-se no momento um problema de saúde que poderá ser ainda maior (apud RODRIGUES, 2010). Complementa Carboni (2007) que esse grupo etário requer uma atenção diferenciada de diversos setores da sociedade, em especial da equipe envolvida nos cuidados com a saúde. Tal necessidade se deve as diversas transformações que podem ocorrer no processo de envelhecimento, esse processo pode ser classificado em primário, secundário e terciário. No envelhecimento primário refere-se às mudanças universais com a idade numa determinada espécie ou populações não sendo dependente de influências ambientais e doença. No envelhecimento secundário ele é resultante das interações das influências externas, e é variável entre indivíduos em meios diferentes. Já no envelhecimento terciário ou terminal é caracterizado por profundas 
perdas físicas e cognitivas, ocasionadas pelo acumular dos efeitos do envelhecimento, como também por patologias dependentes da idade ( FECHINE e TROMPIERE, 2012)

Em relação à qualidade de vida a mesma esta relacionada ao grau de satisfação na vida familiar, amorosa, social e ambiental, e à própria estética existencial, elementos estes que a sociedade considera como padrão de conforto e bem estar, variando com a época, os valores, os espaços e as diferentes histórias, com foco na promoção da saúde que influencia diretamente na vida dos idosos (MIRANZI et al., 2008).

Uma realidade com os diversos grupos populacionais é o aumento da expectativa de vida da população, que conseqüentemente há um aumento proporcional das doenças crônico-degenerativas gerando um enorme desafio para a humanidade, considerando uma parte integrante da saúde e visando garantir às pessoas idosas um envelhecer com saúde e qualidade de vida elevada auxiliando as devidas adaptações características da idade ( SIQUEIRA et.al, 2007).

O interesse para a realização de um estudo que visa identificar a qualidade de vida dos idosos surgiu através da observação do número de idosos que freqüentam as unidades de saúde em busca de consultas e medicamentos, levando em consideração que o aumento da expectativa de vida dos mesmos aumentou ao longo do tempo, justifica saber de que maneira estão enfrentando o processo do envelhecimento.

Sendo assim, o objetivo desse estudo foi a verificação na qualidade de vida dos pacientes idosos cadastrados pelo Programa Hiperdia no município de Cacoal- RO, foi analisado o perfil econômico e sócio demográficos de tais pacientes, avaliando-se a qualidade e expectativa de vida desses pacientes através da aplicação do Whoqol-bref, na qual é composto por quatro domínios: psicológico, físico, relações sociais e meio-ambiente.

\section{MATERIAIS E MÉTODOS}

A coleta de dados foi realizada em 44 dias úteis, entre os meses de julho a Agosto 2014, trata-se de um estudo campo de caráter descritivo, transversal, e com abordagem qualitativa, sendo realizada em uma Unidade Básica de Saúde (UBS) na cidade de Cacoal-RO, que possui 1887 idosos cadastrados no sistema de informação até o período da pesquisa, a princípio seria realizada com 330 idosos, utilizando o cálculo de Barbeta (2011), porém só foi possível obter a amostra de 58 idosos, devido à criação do Programa Saúde Não Tem Preço do Ministério da Saúde criado em 2011, o programa fez com que a maioria dos idosos procurassem as farmácias populares para o recebimento dos medicamentos e não mais a UBS como era de costume, diminuindo assim a demanda de idoso no programa HIPERDIA.

Foi solicitada através de um ofício à gerente administrativa da Unidade Básica de Saúde a autorização para a coleta de dados, onde depois de autorizado foi realizado a aplicação dos instrumentos, para idosos com idade igual ou superior a 60 anos cadastrados no Programa do Hiperdia, que eram atendidas na UBS. Este estudo teve aprovação do Conselho de Ética e Pesquisa (CEP) da Faculdade de Ciências Biomédicas de Cacoal (FACIMED), protocolo ํㅜ 709.425. Foi realizado a abordagem dos idosos que se encontravam na unidade em busca de medicamentos e insumos, onde foram informados sobre os objetivos e procedimentos da pesquisa, sendo convidados a participar da mesma. Aos idosos que consentiram participar da pesquisa, foi oferecido o termo de consentimento livre e esclarecido (TCLE) para assinarem uma das duas vias, uma para o pesquisador e outra para o participante no sentido de consentir sua participação.

A coleta de dados procedeu em dois momentos: primeiro foi aplicado um questionário adaptado da pesquisadora Silva (2007), contendo variáveis relacionadas ás condições socioeconômicas, perfil clinico, educação em saúde e no segundo momento foi à entrevista através da aplicação dos instrumentos WHOQOLbrefque é composto por 26 questões, sendo composta por quatro domínios: Físico, Psicológico, Relações Sociais e Meio Ambiente.

Segundo (KHOURY e NEVES, 2014) o WHOQOL-old consiste em 24 itens da escala de Likert atribuídos a seis facetas: "Funcionamento do Sensório" (FS) avaliando o funcionamento sensorial e o impacto da perda das habilidades sensoriais na qualidade vida, "Autonomia" (AUT) descreve até que ponto se é capaz de viver 
de forma autônoma e tomar as próprias decisões, "Atividades Passadas, Presentes e Futuras" (PPF) referese à satisfação sobre conquistas na vida e coisas a que se anseia, "Participação Social" (PSO) delineia a participação em atividades do cotidiano, especialmente na comunidade, "Morte e Morrer" (MEM) relaciona-se a preocupações, inquietações e temores sobre a morte e o morrer e "Intimidade" avalia a percepção de sentirse amado e apoiado, assim como de amar.

Cada domínio do instrumento WHOQOL-bref é composto de cinco alternativas e cada uma possui valores de 1 a 5, os resultados com escore maior que 2,5 indicam melhor qualidade de vida e os abaixo de 2,5 menor qualidade de vida. Para as questões expressas positivamente, pode-se aplicar a classificação acima. Para as questões expressas negativamente, o escore tem que ser recodificado. No WHOQOL-bref de acordo o manual é necessário recodificar as questões 1, 2, 3, 4 e 26 e no WHOQOL-old as questões 1, 2, 6, 7, 89 e 10 que correspondem aos respectivos valores: $(1=5),(2=4),(3=3),(4=2)$ e $(5=1)$. Foram excluídos idosos com Alzheimer, AIDS, insuficiência renal crônica (com hemodiálise) e outras doenças crônicas; pacientes que foram a óbito ou transferidos para outras unidades e/ou municípios.

Este estudo teve aprovação do Conselho de Ética e Pesquisa (CEP) da Faculdade de Ciências Biomédicas de Cacoal (FACIMED), protocolo oㅜ 709.425. Após a coleta dos dados, os mesmos foram analisados por tabulação usando percentual, realizado com auxílio dos programas word e excel 2010, onde os resultados estão expostos em representações na forma de tabelas e por meio de análise estatísticas (frequências absolutas e relativas).

\section{RESULTADOS E DISCUSSÃO}

No que se refere a caracterização dos participantes da pesquisa, é possível observar que conforme descrito na Tabela 1, as características sociodemográficas dos idosos cadastrados no Programa Hiperdia de uma determinada UBS no município de Cacoal- RO, dos 58 idosos estudados, 31 (67,2\%) são do gênero feminino e $18(32,8 \%)$ do gênero masculino. A faixa etária com maior número de idosos foi entre 60 a 64 anos. Em relação à situação conjugal, com companheiro 34 (58,6\%), viúvo 19 (32,8\%) e separado 5 (8,6\%). Quanto à escolaridade a maior porcentagem foi de idosos com ensino fundamental incompleto de 31 (53,4\%), seguido do analfabetismo 24 (41,5\%) e ensino médio 3 (5,2\%). São aposentados ou pensionistas 52 (89,7\%). A renda mensal da maioria dos idosos é de 1 a 2 salários mínimos no total de 46 (79,3\%). Quando indagadas se tinham alguma limitação física $12(20,7 \%)$ responderam que sim e $46(79,3 \%)$ responderam que não.

Avaliando a segunda tabela referente ao domínio físico que está relacionado a dor e desconforto, energia e fadiga, sono e repouso, atividades da vida cotidiana, dependência de medicação ou de tratamentos e capacidade de trabalho. Quando questionados sobre em que medida a sua dor física o impedia de fazer o que precisava, 49 idosos responderam nada ou muito pouco, na pergunta sobre se precisava de algum tratamento médico para levar sua vida diária 33 responderam que precisava bastante ou extremamente, com isso justifica-se o porquê da Tabela 1 apresentar um índice baixo de limitação física, pois os idosos pesquisados são portadores de Hipertensão e Diabetes dependentes de medicação, embora por se tratar de doenças crônicas que podem até levar a um acometimento físico, os idosos da presente pesquisa não se consideram portadores de limitação física. O estudo de Oliveira (2012) constatou resultado semelhante, $36,2 \%$ dos pesquisados relatam que a dor nada impede de fazer o que precisam, $32,5 \%$ muito pouco.

O domínio psicológico que se refere a sentimentos positivos e negativos, pensar, aprender, memória e concentração, auto-estima, imagem corporal e aparência, espiritualidade, crenças pessoais e religiosidade, apresentou relevância estatística em nível de satisfação com média 3,7, pois os idosos estão bastante ou extremamente satisfeitos consigo mesmo, em relação a sentimentos negativos como mau humor, desespero, ansiedade e depressão, os mesmos relataram nunca ou algumas vezes ter esse tipo de sentimento. Através das respostas obtidas, constatou-se que a maioria dos idosos não apresentaram comprometimentos com sua qualidade de vida, observadas pelos domínios expostos. 
Tabela 1 - Característica sociodemográfica dos idosos cadastrados no Programa Hiperdia de uma UBS no Munícipio de Cacoal/RO, 2014.

\begin{tabular}{|c|c|c|}
\hline VARIÁVEIS & $\mathrm{N}$ & $\%$ \\
\hline \multicolumn{3}{|c|}{ Perfil Sócio Demográfico e Econômico } \\
\hline \multicolumn{3}{|l|}{ Gênero } \\
\hline Masculino & 19 & 32,8 \\
\hline Feminino & 39 & 67,2 \\
\hline \multicolumn{3}{|l|}{ Faixa Etária } \\
\hline $60-64$ & 21 & 36,2 \\
\hline $65-69$ & 14 & 24,1 \\
\hline $71-76$ & 13 & 22,4 \\
\hline $77-81$ & 6 & 10,4 \\
\hline $83-86$ & 4 & 6,9 \\
\hline \multicolumn{3}{|l|}{ Situação Conjugal } \\
\hline Com companheiro & 34 & 58,6 \\
\hline Viúvo & 19 & 32.8 \\
\hline Separado & 5 & 8,6 \\
\hline \multicolumn{3}{|l|}{ Escolaridade } \\
\hline Ensino Fundamental incompleto & 31 & 53,4 \\
\hline Ensino Médio & 3 & 5,2 \\
\hline Não Alfabetizado & 24 & 41,4 \\
\hline \multicolumn{3}{|l|}{ Aposentadoria/Pensionista } \\
\hline $\begin{array}{l}\text { Sim } \\
\text { Não }\end{array}$ & $\begin{array}{c}52 \\
6\end{array}$ & $\begin{array}{l}89,7 \\
10,3\end{array}$ \\
\hline \multicolumn{3}{|l|}{ Renda Mensal } \\
\hline 1 a 2 Salário Mínimo & 46 & 79,3 \\
\hline 3 a 4 Salário Mínimo & 11 & 19 \\
\hline 5 a 6 Salário Mínimo & 1 & 1,7 \\
\hline \multicolumn{3}{|l|}{ Limitação Física } \\
\hline Sim & 12 & 20,7 \\
\hline Não & 46 & 79,3 \\
\hline Total & 58 & 100 \\
\hline
\end{tabular}

Fonte: Dados da pesquisa,2014.

Na Tabela 2 demonstra os dados descritivos dos domínios de qualidade de vida percebidos pelos idosos cadastrados no programa Hiperdia de uma Unidade Básica de Saúde do Município de Cacoal/RO, 2014 referente ao instrumento WHOQOL-BREF GERAL, ao analisar os diferentes domínios do questionário WHOQOL-BREF, o domínio que mais se destacou foi o social com média de $(3,8)$, seguido do psicológico $(3,7)$, ambiental $(3,4)$ e físico $(3,3)$. Situação é igual encontrada no estudo Serbim e Figueiredo (2011), no qual o domínio que mais contribuiu na qualidade de vida dos idosos foi o social, seguido do ambiental, psicológico e físico. Em relação à participação social a qual se avalia ao nível de atividade do idoso (individuais ou comunitárias), de maneira com a qual o idoso utiliza seu tempo, o presente estudo constatou referente a este domínio uma percepção positiva sobre a qualidade de vida. 
Tabela 2- Dados descritivos dos domínios de qualidade de vida percebidos pelos idosos cadastrados no programa Hiperdia de uma Unidade Básica de Saúde do Município de Cacoal/RO, 2014 referente ao instrumento WHOQOL-BREF GERAL.

\begin{tabular}{lcc}
\hline \multicolumn{1}{c}{ VARIÁVEL } & MÉDIA & DESVIO PADRÃO \\
\hline Domínio Físico & 3,3 & 0,5 \\
Domínio Psicológico & 3,7 & 0,5 \\
Domínio Social & 3,8 & 0,5 \\
Meio Ambiente & 3,4 & 0,4 \\
\hline
\end{tabular}

Fonte :Dados da pesquisa,2014.

A tabela 3 constata a percepção através do instrumento WHOQOL-BREF da qualidade de vida (Q1) e satisfação com saúde (Q2) dos idosos cadastrados no Programa Hiperdia de uma Unidade Básica de Saúde do Município de Cacoal/RO, corresponde ás duas perguntas gerais do WHOQOL-bref sobre qualidade de vida questão 1 (Q1) "Como você avalia sua qualidade de vida" e questão 2 (Q2) "Quão satisfeito (a) você está com a sua saúde". Foi certificado entre os entrevistados em relação à percepção da qualidade de vida, que cerca de 3,6 afirmaram que sua qualidade de vida é boa ou muito boa e que aproximadamente 3,3 afirmaram que estão satisfeitos ou muito satisfeitos com a sua saúde.

Tabela 3 - Percepção através do instrumento WHOQOL-BREF da qualidade de vida (Q1) e satisfação com saúde (Q2) dos idosos cadastrados no Programa Hiperdia de uma Unidade Básica de Saúde do Município de Cacoal/RO, 2014.

\begin{tabular}{ccc}
\hline QUESTÃO & MÉDIA & DESVIO PADRÃO \\
\hline Questão1-Como você avaliaria sua qualidade de vida? & 3,6 & 0,8 \\
Questão2-Quão satisfeito (a) você está com sua saúde? & 3,3 & 0,8 \\
\hline
\end{tabular}

Fonte: Dados da pesquisa,2014.

A qualidade de vida é um conceito subjetivo, pelo qual ninguém, exceto o próprio indivíduo, pode relatar como ele percebe da mesma. De acordo com o estudo realizado por Oliveira et al. (2012), onde verificou-se que $84 \%$ dos idosos avaliam sua qualidade de vida como boa e muito boa e a maior parte dos idosos afirmaram que estão satisfeitos, apresentando um nível melhor de qualidade de vida.

A autonomia é um determinante básico para uma boa qualidade de vida, a média de 3,5 considera os pesquisados satisfeitos. A autonomia dos idosos reflete diretamente na sua participação social, pois o capacita a participar de um processo no qual interagem aspectos externos e internos a sua escolha. Os estudos Nunes et al. (2010), Dalsentere Matos (2009), Tavareset al. (2011), apresentaram resultados semelhantes. A autonomia aborda a percepção do indivíduo sobre a sua liberdade para tomar suas próprias decisões e gerir sozinho seu próprio estilo de vida.

$\mathrm{Na}$ Tabela 4 destaca os dados descritivos das facetas referentes ao questionário de Qualidade de vida WHOQOL-OLD GERAL, dos idosos cadastrados no Programa Hiperdia de uma Unidade Básica de Saúde do município de Cacoal/RO, Nas facetas autonomia e participação social, foram encontradas um escore médio de 3,5 obtendo a média mais baixa que as demais facetas, entretanto nas facetas sensório, Atividade Presente, Passada e Futura e geral apresenta um escore médio de 3,7 sendo um intermédio entre as facetas. E nas facetas morte e morrer, intimidade o escore é de 3,9 indicando que os idosos desse estudo estão satisfeitos quanto aos sentimentos de companheirismo e amor, também em relação às inquietações e temores com a morte e morrer, este fato está interligado um com o outro, pois o idoso intimamente insatisfeito, conseqüentemente terá menos medo da morte. Correlacionando com o estudo de Nuneset al. (2010), onde afirma que pode estar relacionado ao fato, de que, por se sentirem no final da vida a morte significa algo já esperado por eles. 
Tabela 4 - Dados descritivos das facetas referentes ao questionário de Qualidade de vida WHOQOL-OLD GERAL, dos idosos cadastrados no Programa Hiperdia de uma Unidade Básica de Saúde do município de Cacoal/RO, 2014.

\begin{tabular}{lcc}
\hline \multicolumn{1}{c}{ VARIÁVEL } & MÉDIA & DESVIO PADRÃO \\
\hline Sensório & 3,7 & 0,7 \\
Autonomia & 3,5 & 0,5 \\
Atividade Presente, Passada e Futura & 3,7 & 0,5 \\
Participação Social & 3,5 & 0,4 \\
Morte e Morrer & 3,9 & 0,9 \\
Intimidade & 3,9 & 0,5 \\
Geral & 3,7 & 0,3 \\
\hline
\end{tabular}

Fonte: Dados da pesquisa,2014

A morte constitui um processo natural da existência humana, juntamente com o envelhecer, porém nem sempre são aceitos pelos seres que o vivenciam. Quando há uma valorização do saber e da história de vida de cada idoso, quando estes são entendidos e respeitados, ele percebe que sua existência tem um significado. Considerar, respeitar e amar permite o acolhimento ao idoso, o reconhecimento de sua singularidade, promovendo um sentido no envelhecer e possibilitando o enfrentamento da morte (FALLER, 2010). O domínio intimidade aborda os sentimentos de companheirismo e amor em uma relação a um companheiro ou pessoa próxima.

\section{CONCLUSÃO}

Perante os resultados obtidos, acredita-se que o presente estudo trará contribuição significativa ao planejamento das ações em saúde voltadas a qualidade de vida dos idosos cadastrado no programa Hiperdia no município de Cacoal - RO, pois permitiu a compreensão adequada sobre os entrevistados e sugere que as entidades sejam sensibilizadas para desenvolver e apoiar iniciativas que visam melhorar a qualidade de vida deste grupo.

É necessário que estudos da qualidade de vida dos idosos ocupem lugar de destaque, uma vez que os avanços na área da saúde contribuíram para o aumento na expectativa de vida e no contingente de idosos. Portanto, o envelhecimento populacional em nosso país já é uma realidade e ações que busquem lidar com esse crescente contingente de idosos devem ser priorizadas em todas as áreas do saber. As universidades e instituições têm como papel social prestar a sua contribuição, desenvolvendo e apoiando iniciativas que visem melhorar a qualidade de vida dos idosos.

\section{REFERÊNCIAS}

1. BARBETTA, Pedro Alberto. Estatística Aplicada Às Ciências Sociais, $7^{a}$ edição, 2011.

2. BRAGA, Maria Cecilia Portugal, et al.,Qualidade De Vida Medida Pelo Whoqol-Bref: Estudo Com Idosos Residentes Em Juiz De Fora/Mg, 2011. Rev. APS; 2011; jan/mar; 14(1); 93-100.

3. BRASIL, Caderno de Atenção Básica Diabetes Mellitus, 2006 a. BRASIL, Caderno de Atenção Básica Hipertensão Arterial Sistêmica(HAS), 2006 b.

4. CARBONI, Rosadélia Malheiros; REPPETTO, Maria Ângela. Uma reflexão sobre a assistência à saúde do idoso no Brasil . Revista Eletrônica de Enfermagem, v. 09, n. 01, p. 251 - 260, 2007.

5. DALSENTER, Claudia Araci; MATOS, Fabio Marcelo. Percepção da Qualidade de Vida Em Idosos Institucionalizados da Cidade de Blumenau, Sc, 2009. Dynamis revista tecno-cientifica (2009) n.2 vol.15, 32-37.

6. FALLER, JossianaWilkeet al., Qualidade De Vida De Idosos Cadastrados Na Estratégia Saúde Da Família De Foz Do Iguaçu-Pr, 2010. Escola Anna Nery, 14(4), 803-810. 
7. FECHINE, Basílio Rommel Almeida; TROMPIERI, Nicolino. O processo de envelhecimento: as principais alterações que acontecem com o idoso com o passar dos anos. Revista Cientifica Internacional. Edição 20, volume 1, artigo no 7, Janeiro/Março 2012.

8. FELICIANO, A B; MORAES, S A; FREITAS, I C M; O perfil do idoso de baixa renda no Município de São Carlos, São Paulo, Brasil: um estudo epidemiológico, 2004. Cadernos de Saúde Pública, 20(6), 1575-1585.

9. INOUYE, Keika; PEDRAZZANI, Elisete Silva. Nível de instrução, status socioeconômico e avaliação de algumas dimensões da qualidade de vida de octogenários, 2007. Revista Latino-Americana de Enfermagem, 15(spe), 742-747.

10. KHOURY, Hilma Tereza Tôrres, \& SÁ-NEVES, Ângela Carina. Percepção de controle e qualidade de vida: comparação entre idosos institucionalizados e não institucionalizados. Revista Brasileira de Geriatria e Gerontologia, 17(3), 553-565.

11. MIRANZI, Sybelle de Souza Castro. et al., Qualidade De Vida De Indivíduo Com Diabetes Mellitus E Hipertensão Acompanhados Por Uma Equipe De Saúde Da Família, 2008. Texto \& Contexto - Enfermagem, 17(4), 672-679.

12. NUNES, Vilani Medeiros de Araújo; MENEZES, Rejane Maria Paiva; ALCHIERI, João Carlos. Avaliação da Qualidade de Vida em idosos institucionalizados no município de Natal, Estado do Rio Grande do Norte, 2010. Acta Scientiarum V.32, n.2 (2010).

13. ORGANIZAÇÃO MUNDIAL DE SAÚDE. Envelhecimento Ativo: Um Política De Saúde, 2005.

14. RODRIGUES, Suely Maria. Saúde Bucal e Qualidade De Vida Doidoso Usuário Do Sistema Único De Saúde De Um Município De Médioporte Em Minas Gerais - Brasil, 2010. Dissertação ( Doutorado em Odontologia), Universidade Federal de Minas Gerais, 2010.

15. SERBIM, A.K.; FIGUEIREDO, A.E.P. L. Qualidade de vida de idosos em um grupo de convivência, Scientia Medica. v.21, 2011.

16. SILVA, Telma Pereira Vieira. Qualidade de vida dos idosos da cidade de Rio Verde-GO, 2007. Dissertação ( Mestrado em Ciências da Saúde), Universidade de Brasília, 2007.

17. SIQUEIRA, Fernando V, Facchini, Luiz Augusto, Piccini, Roberto X, Tomasi, Elaine, Thumé, Elaine, Silveira, Denise S, Vieira, Vera, \& Hallal, Pedro C. Prevalência de quedas em idosos e fatores associados. Revista de Saúde Pública, 41(5), 749-756.

18. TAVARES, Darlene Mara dos Santos, et.al., Qualidade de Vida de Idosos com e sem Hipertensão Arterial. Rev. Eletr. Enf. [Internet]. $2011 \mathrm{abr} / \mathrm{jun} ; 13(2): 211-8$.

19. THE WORLD HEALTH ORGANIZATION QUALITY OF LIFE. Disponível em: $<$ http://www.cefid.udesc.br/arquivos/id submenu/1173/whoqol bref.pdt>. Acesso em: 16 de agosto de 2014 as 15:29 h.

20. VERAS, Renato. Envelhecimento populacional contemporâneo: demandas, desafios e inovações. Revista de Saúde Pública, 43(3), 548-554. Epub April 17, 2009.

21. ZASLAVSKY, Cláudio. GUS, Iseu. Idoso: Doença Cardíaca e Comorbidades. Arquivos Brasileiros de Cardiologia, 79(6), 635-639. 